Check for updates

Cite this: RSC Adv., 2018, 8, 30919

\title{
SKLB023 as an iNOS inhibitor alleviated liver fibrosis by inhibiting the TGF-beta/Smad signaling pathway
}

\author{
Jinhang Zhang, $\dagger^{\mathrm{a}}$ Yanping Li, $\hat{\dagger}^{\mathrm{a}}$ Qinhui Liu, ${ }^{\mathrm{a}}$ Rui Li, ${ }^{\mathrm{a}}$ Shiyun Pu, ${ }^{a}$ Lina Yang, ${ }^{\mathrm{b}}$ \\ Yanhuan Feng ${ }^{\mathrm{b}}$ and Liang $\mathrm{Ma}$ (D) *b
}

\begin{abstract}
Nonalcoholic steatohepatitis (NASH)-related liver fibrosis has been suggested to be a physiological consequence of chronic hepatic injury, necrosis, inflammation and unbalanced intrahepatic lipid metabolism. Accumulated evidence demonstrates that inducible nitric oxide synthase (iNOS) is highly expressed in advanced liver fibrosis, and the knockout of iNOS inhibits the progression of hepatic fibrosis. In our previous study, (Z)-N-(3-chlorophenyl)-2-(4-((2,4-dioxothiazolidin-5-ylidene)methyl) phenoxy)acetamide (SKLB023), a novel small-molecule inhibitor of iNOS, blocked joint inflammation and cartilage destruction in arthritis. However, the role and function of SKLBO23 in liver fibrosis have not been fully elucidated. In the present study, methionine- and choline-deficient (MCD) diet-induced NASH mice and LX-2 hepatic stellate cells were chosen to investigate the pharmacological effects of SKLB023 against liver fibrosis and the associated mechanism. Our results show that SKLB023 significantly alleviated MCD diet-induced liver injury, lipid accumulation and liver fibrosis. SKLB023 could suppress the activation of hepatic stellate cells by interfering with TGF- $\beta /$ Smad pathways. Importantly, SKLB023 inhibited the level of TGF- $\beta 1$ and Smad2/3 phosphorylation by blocking the expression of iNOS. These results suggest that SKLB023 might be an effective drug candidate for the treatment of liver fibrosis.
\end{abstract}

Received 11th June 2018

Accepted 4th August 2018

DOI: $10.1039 / \mathrm{c} 8 \mathrm{ra04955f}$

rsc.li/rsc-advances
The initiation of chronic liver diseases commonly involves an inflammatory phase, which progresses to fibrosis after continuous oxidative stress. ${ }^{7}$ Under these conditions, inducible nitric oxide synthase (iNOS) is upregulated, leading to the production of large amounts of nitric oxide (NO). ${ }^{8}$ Since NO is reported to negatively regulate diverse HSC functions that lead to its activation, excessive NO production may promote the development of liver fibrosis. ${ }^{9,10}$ In liver fibrosis patients, mild liver fibrosis displayed a faint and positive relationship with the iNOS expression, while the expression of iNOS was higher in those with advanced fibrosis. ${ }^{11,12}$ iNOS knockout mice fed a high-cholesterol diet for six weeks exhibited a significant reduction in hepatic fibrosis. ${ }^{13}$ Furthermore, the iNOS inhibitor FR260330 improved liver fibrosis in rats after oral administration. ${ }^{14}$ These results indicate that iNOS plays crucial roles in the process of liver fibrosis, and the pharmacological inhibition of iNOS shows promise for the treatment of liver fibrosis.

\footnotetext{
${ }^{a}$ Laboratory of Clinical Pharmacy and Adverse Drug Reaction, Department of Pharmacy, Collaborative Innovation Centre of Biotherapy, West China Hospital of Sichuan University, Chengdu 610041, China

${ }^{b}$ Kidney Research Lab, Division of Nephrology, West China Hospital of Sichuan University, Chengdu 610041, China. E-mail: Liang_m@scu.edu.cn; Fax: +86-2885423341; Tel: $+86-28-85164167$

$\dagger$ These authors contributed equally to this work.
}<smiles>O=C(COc1ccc(/C=C2\SC(=O)NC2=O)cc1)Nc1cccc(Cl)c1</smiles>

Fig. 1 Chemical structure of SKLB023. 
(Z)- $N$-(3-Chlorophenyl)-2-(4-((2,4-dioxothiazolidin-5-ylidene) methyl)phenoxy)acetamide (SKLB023, Fig. 1), is a novel, chemically synthesized iNOS inhibitor based on the thiazolidine-2,4dione moiety. ${ }^{15}$ In our previous studies, SKLB023 was found to exhibit excellent anti-inflammatory activity in arthritis and antifibrotic effects in renal interstitial fibrosis. ${ }^{\mathbf{1 6 , 1 7}}$ However, the efficacy of SKLB023 in chronic liver diseases remains undetermined. Thus, in the present study, we use methionine/cholinedeficient (MCD) diet-induced NASH mice and LX-2 cells to investigate the in vivo and in vitro inhibition of liver fibrosis by SKLB023, respectively, and the associated mechanism.

\section{Methods}

\section{Animal model and treatment}

C57BL/6J male mice were purchased from Sichuan University Laboratory Animal center (Chengdu, China). The mice were housed with a $12: 12$ hours light/dark cycle and permitted ad libitum consumption of water and diet. For the NASH model, eight-week-old mice received a MCD diet (TrophicDiet, China) or an amino acid diet (TrophicDiet, China) as a control for six weeks. After diet feeding for four weeks, the mice were treated with saline or SKLB023 (25 mg per kg per day) for two weeks by gavage daily. At the end of diet treatment, all mice were sacrificed, and blood and liver samples were collected and stored for further analysis. Animal experiments were approved by the Animal Ethics Committee of West China Hospital, Sichuan University (No. 2016-273).

\section{Serum alanine transaminase (ALT) and aspartate aminotransferase (AST) assays}

Blood was held at $4{ }^{\circ} \mathrm{C}$ for $1 \mathrm{~h}$ and then centrifuged at $3000 \mathrm{rpm}$ for $10 \mathrm{~min}$. Serum was collected from the supernatant and stored at $-80{ }^{\circ} \mathrm{C}$. Serum ALT and AST levels were measured by appropriate enzymic kits from Biosino (Beijing, China).

\section{Hepatic lipid analysis}

Liver samples (150 mg) were homogenized with $1 \mathrm{ml}$ PBS for $5 \mathrm{~min}$. The homogenates were added to $2.5 \mathrm{ml}$ chloroform/ methanol (2:1) and centrifuged at $3000 \mathrm{rpm}$ for $10 \mathrm{~min}$. The lower phase was transferred to a new tube. An additional $1 \mathrm{ml}$ of solvent was added to the upper phase and centrifuged. The lower phase was collected and combined with washing buffer. After centrifugation, the lower phase containing lipids was evaporated. The dried pellet was resuspended in 1\% Triton X100 in ethanol and analyzed using total cholesterol and triglycerides kits (Biosino, Beijing, China).

\section{Histological staining}

Livers were fixed in $10 \%$ formalin, embedded in paraffin, sectioned at $4 \mu \mathrm{m}$, and stained with hematoxylin and eosin (H\&E) or Sirius Red. Frozen sections $(6 \mu \mathrm{m})$ were used for Oil Red O staining. Images were captured by a microscope (Nikon, Tokyo, Japan).

\section{Cell culture and treatments}

The human HSC line LX-2 was provided by Prof. Lieming Xu from Shuguang Hospital, Shanghai University of Traditional Chinese Medicine. The LX-2 cells were cultured in Dulbecco's modified Eagle's medium (DMEM; Gibco, USA) supplemented with $10 \%$ fetal bovine serum (FBS; Hyclone, USA) and incubated at $37{ }^{\circ} \mathrm{C}$ with $5 \% \mathrm{CO}_{2}$. To test the effect of SKLB023 on TGF- $\beta 1 /$ Smad signaling, the cells were exposed to TGF- $\beta 1$ (2 $\mathrm{ng} \mathrm{ml}{ }^{-1}$; Peprotech, USA) and treated with SKLB023 for $48 \mathrm{~h}$.

\section{RT-PCR analysis}

Total hepatic RNA was extracted with Trizol reagent and reversetranscribed into complementary DNA (RR037A; TaKaRa, Kyoto, Japan). Quantitative real-time PCR involved the SYBR greenbased assay with the CFX96 Real-Time system (Bio-Rad, Hercules, CA). The primer sequence was as follows: $\alpha$-SMA, sense $\left(5^{\prime}-3^{\prime}\right)$ CAGGGAGTAATGGTTGGAAT, anti-sense $\left(5^{\prime}-3^{\prime}\right)$ TCTCAAACATAATCT GGGTCA; Col1a1, sense $\left(5^{\prime}-3^{\prime}\right)$ CATGAGCCGAAGCTAACCC, anti-sense $\left(5^{\prime}-3^{\prime}\right)$ TGTGGCAGATACAGATCAAGC; Col3a1, sense $\left(5^{\prime}-3^{\prime}\right)$ TGCTCCTGTGCTTCCTGATG, anti-sense $\left(5^{\prime}-3^{\prime}\right)$ GACCTGGTTGTCCTGG AAGG; Timp-1, sense $\left(5^{\prime}-3^{\prime}\right)$ GCAACTCGGACCTGGTCATAA, anti-sense $\left(5^{\prime}-3^{\prime}\right)$ CGGCCCGTGATGAGAAACT.

\section{Western blot analysis}

Liver samples were lysed in lysis buffer and subjected to western blot analysis with the following primary antibodies: $\alpha$-SMA (Sigma, USA), Col1a1 (Boster, China), iNOS (Abcam, USA), TGF$\beta 1$ (Abcam, USA) t-Smad2/3 (CST, USA), p-Smad2/3 (CST, USA) and $\beta$-tubulin (Zhengneng, China). Blots were visualized using a LI-COR (Lincoln, NE) Odyssey System. Quantitative determination of band intensity was conducted using Image Studio analysis software version 4.0 (LI-COR).

\section{Statistical analysis}

Data are expressed as the mean \pm SEM for each sample. Statistical significance was determined by Student's unpaired twotailed $t$ test or one-way ANOVA for multiple comparisons with Tukey's test. $P<0.05$ was considered statistically significant.

\section{Results and discussion}

\section{SKLB023 alleviates steatosis in MCD diet-induced mice}

The most widely used diet to induce NAFLD/NASH is the MCD diet, which results in liver injury histologically similar to human NASH. ${ }^{18}$ The standard MCD diet has a high content of sucrose (40\% of energy) and is moderately enriched with fat (10-20\%). It is a very reproducible model that consistently induces the phenotype of severe NASH, including steatosis, hepatic inflammation and fibrosis after 6-8 weeks of administration.

To determine the effect of SKLB023 on NASH, we fed mice with an amino acid control diet or the MCD diet for six weeks. ALT and AST are surrogate markers for liver injury. As expected, the MCD diet significantly increased the serum levels of ALT and AST compared to the control diet (Fig. 2A and B). The 


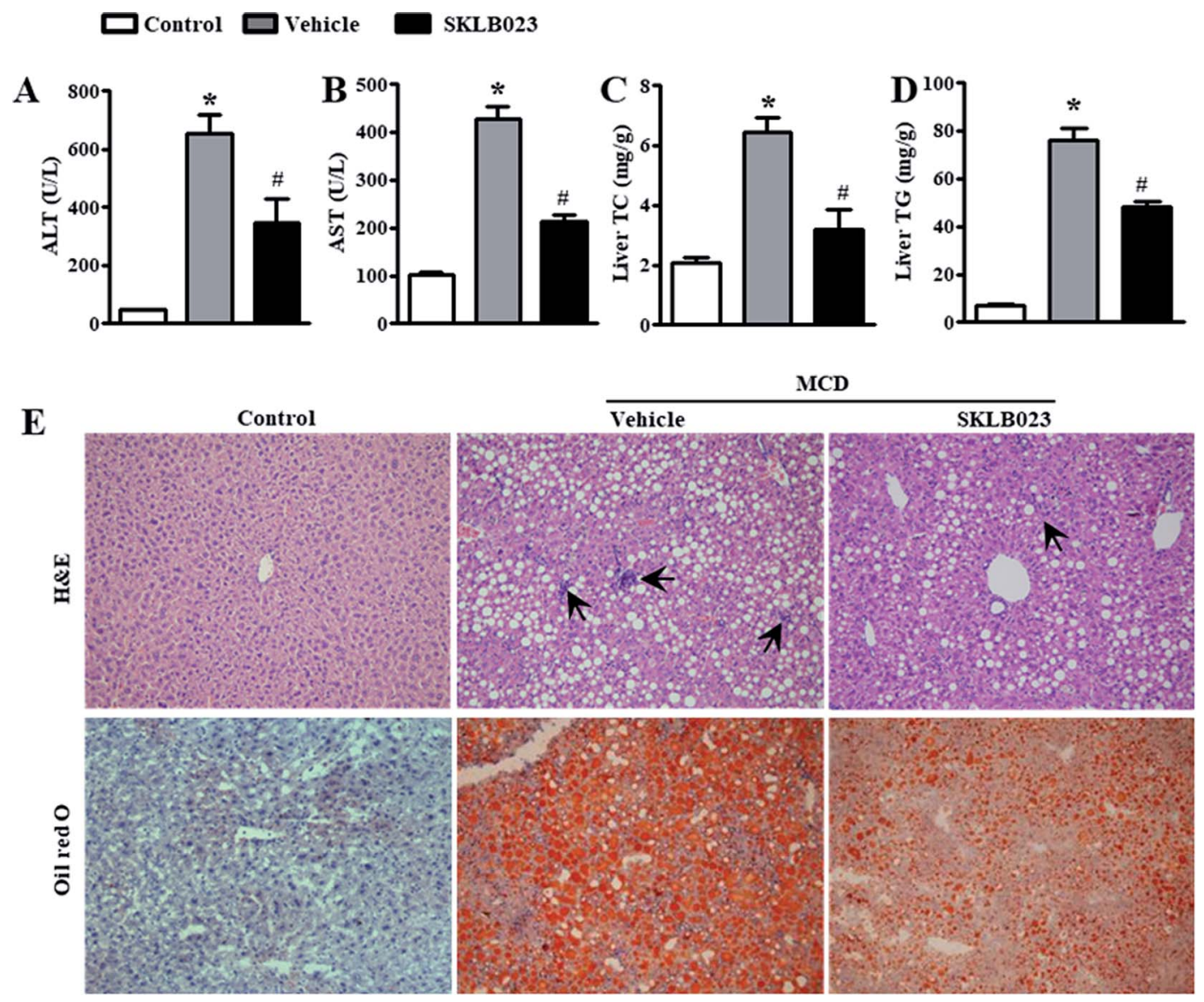

Fig. 2 SKLB023 alleviates steatosis in MCD diet-induced mice. Serum levels of (A) ALT and (B) AST; hepatic levels of (C) total cholesterol (TC) and (D) triglyceride (TG); and (E) representative H\&E staining and Oil Red $O$ staining of liver sections of mice with indicated treatments. $* P<0.05$ versus the control group, ${ }^{\#} P<0.05$ versus the vehicle, $n=6$ for each group.

elevation of serum ALT and AST was suppressed by treatment with SKLB023 at a dose of $25 \mathrm{mg} \mathrm{kg}^{-1}$ day $^{-1}$ for two weeks. Meanwhile, the MCD diet significantly increased the levels of hepatic triglyceride (TG) and cholesterol (TC) compared to the control diet (Fig. 2C and D). The elevation of hepatic TG and TC was also inhibited by the oral administration of SKLB023.

Histological examination also provided evidence supporting the results of the serum biochemical analysis. As shown in Fig. 2E, MCD diet-treated mice had obvious vesicular lesions and severe neutrophil infiltration. In animals treated with SKLB023, significant reductions were observed in vacuoles and inflammation. Consistently, PBMM/SKLB023 exhibited greatly reduced fat droplets compared to free SKLB023, as shown by oil red staining. These results indicate that treatment with SKLB023 prevented MCD diet-induced liver injury and lipid accumulation.

\section{SKLB023 alleviates hepatic fibrosis in MCD diet-induced mice}

Hepatic fibrosis is a dynamic process characterized by the net accumulation of ECM resulting from NASH. ${ }^{4}$ The assessment of liver fibrosis indicated that the MCD diet induced collagen deposition, as shown by Sirius Red staining (Fig. 3A). SKLB023 treatment reduced the collagen deposition. To investigate if
SKLB023 suppressed the activation of HSCs in vivo, we examined the hepatic mRNA expression of activation markers. As shown in Fig. 3B, the mRNA expressions of $\alpha$-SMA, collagen and Timp-1 were elevated considerably by the MCD diet. Treatment with SKLB023 resulted in significant decreases in the mRNA expressions of the corresponding fibrosis-related genes. The reduced protein expression levels of $\alpha$-SMA and Col1a1 in SKLB023-treated mice were further confirmed by western blotting (Fig. 3C). Hence, these data indicate that SKLB023 can inhibit liver fibrosis in vivo.

\section{SKLB023 inhibits hepatic TGF- $\beta 1$ and Smad2/3 phosphorylation}

Excess iNOS-induced NO was implicated in the etiologies of liver fibrosis. ${ }^{8}$ Given the beneficial roles of iNOS inhibition and gene deletion in liver fibrosis observed in experimental models, iNOS is likely a potential therapeutic target for hepatic fibrosis. ${ }^{9,13}$ In this study, the expression of iNOS protein was barely detectable in the control group. The hepatic iNOS protein content was significantly increased in the group treated with MCD diet compared to the control (Fig. 4A). Importantly, the elevation of iNOS was successfully blocked by SKLB023 treatment. 

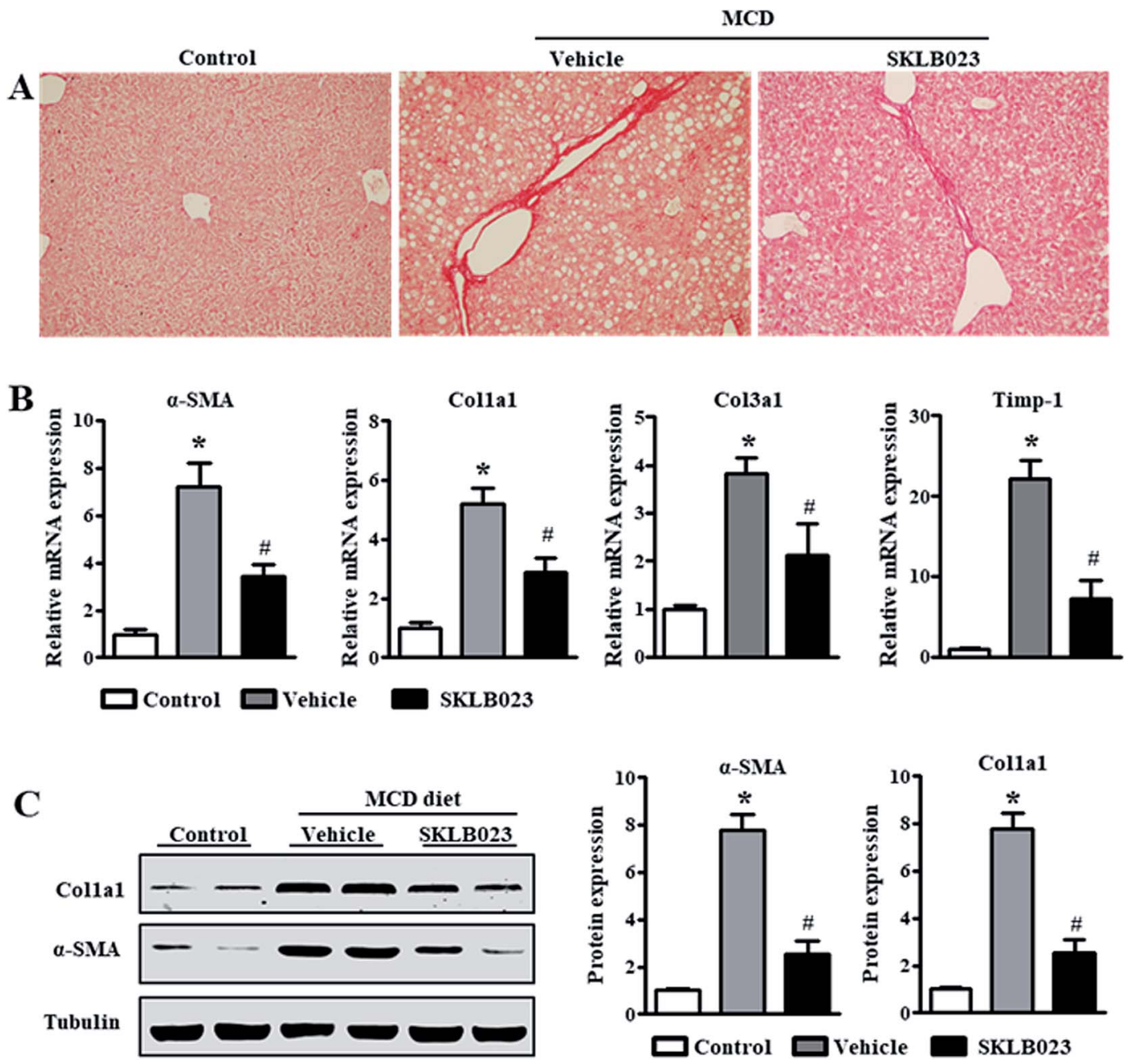

Fig. 3 SKLB023 alleviates hepatic fibrosis in MCD diet-induced mice. (A) Representative images of Sirius Red staining demonstrate hepatic collagen deposition in the indicated group; (B) hepatic mRNA expressions of fibrosis-related genes measured by real-time polymerase chain reaction ( $n=6$ for each group); and (C) protein expression levels of hepatic $\alpha$-SMA and Col1a1 measured by western blot analysis. The relative average protein level was determined by densitometry and normalized with $\beta$-tubulin. ${ }^{*} P<0.05$ versus the control group, ${ }^{\#} P<0.05$ versus the vehicle.

The increase in transforming growth factor- $\beta$ (TGF- $\beta$ ) expression is considered as a key driver in the activation of HSCs to aggravate liver fibrosis. ${ }^{\mathbf{1 9 , 2 0}}$ As shown in Fig. 4B, the expression of TGF- $\beta 1$ protein was markedly induced in the mice model of liver fibrosis. The oral administration of SKLB023 significantly reduced the upregulation of hepatic TGF- $\beta 1$ protein.

Previous studies have confirmed that the TGF- $\beta /$ Smads signaling pathway is the central regulator of HSC activation and the onset of liver fibrosis. ${ }^{21,22}$ The TGF- $\beta /$ Smads pathway is activated by TGF- $\beta$ binding to its receptors. The downstream proteins, namely Smad2 and Smad3, are activated by phosphorylation, which further promotes the transcription of genes responsible for ECM protein synthesis and the progression of liver fibrosis. Western blot analysis indicated that the expression of total Smad2/3 in liver was not affected by MCD diet and SKLB023 (Fig. 4B). However, the MCD diet-induced phosphorylation of Smad2/3 was abolished by the oral administration of SKLB023. Taken together, these results indicate that SKLB023 can inhibit the TGF- $\beta /$ Smad signaling pathways in fibrotic liver.
SKLB023 inhibits the TGF- $\beta 1$-induced activation of hepatic stellate cells

During chronic liver injury, TGF- $\beta$ plays a prominent role in stimulating liver fibrogenesis by myofibroblast-like cells derived from HSCs. To evaluate the potential inhibitory effect of SKLB023 against HSCs, the in vitro inhibitory rate of SKLB023 for LX-2, a well-established HSC cell line, was detected and quantified by CCK-8 assay. As shown in Fig. 5A, the 50\% inhibitory concentration of SKLB023 against LX-2 was 36.06 and $19.48 \mu \mathrm{M}$ after 24 and $48 \mathrm{~h}$, respectively.

To investigate the regulatory effects of SKLB023 in the TGF$\beta 1$-induced activation of LX-2, the expressions of $\alpha$-SMA and Col1a1 were examined by western blot analysis. In Fig. 5B, following treatment with TGF- $\beta 1$, the expressions of $\alpha$-SMA and Col1a1 in LX-2 were dramatically increased compared to the control. SKLB023 treatment significantly decreased the production of $\alpha$-SMA and Col1a1 in a dose-dependent manner in LX-2 compared to in the TGF- $\beta 1$-induced group. 
$\mathbf{A}$
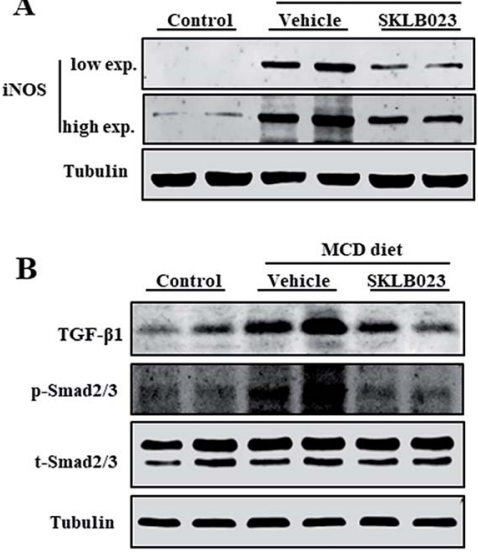
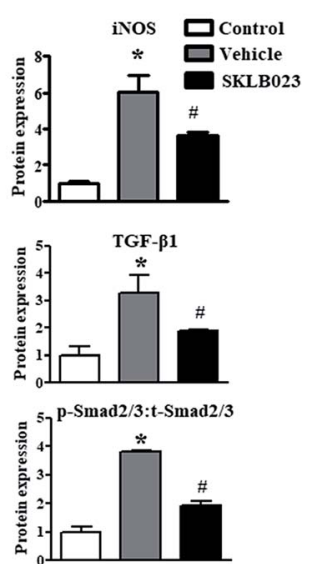

Fig. 4 SKLB023 inhibits Smad2/3 phosphorylation in vivo. The hepatic protein expressions of (A) iNOS, and (B) TGF- $\beta 1$ and $p-S m a d 2 / 3$ were measured by western blot, and the relative average protein level was determined by densitometry and normalized with $\beta$-tubulin or $\mathrm{t}$ Smad2/3, respectively. ${ }^{*} P<0.05$ versus the control group, ${ }^{\#} P<0.05$ versus the vehicle.
A
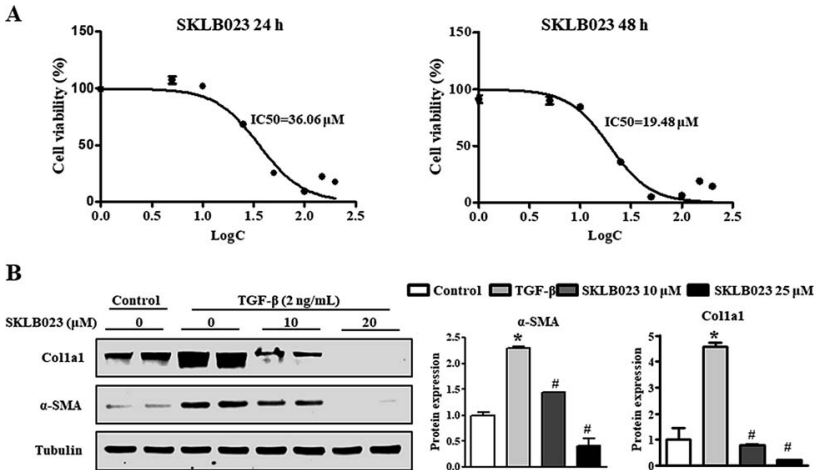

Fig. 5 SKLB023 inhibits the TGF- $\beta 1$-induced activation of hepatic stellate cells. (A) The $50 \%$ inhibitory concentration $\left(\mathrm{IC}_{50}\right)$ of SKLBO23 against LX-2 was determined by CCK- 8 assay. (B) The protein expressions of $\alpha$-SMA and Colla1 were measured by western blot analysis. TGF- $\beta 1$-induced LX-2 cells were treated with SKLB023 (10 and $20 \mu \mathrm{M})$ for $48 \mathrm{~h}$. The relative average protein level of $\alpha$-SMA and Col1a1 was determined by densitometry and normalized with $\beta$ tubulin. ${ }^{*} P<0.05$ versus the control group, ${ }^{*} P<0.05$ versus the vehicle.

\section{SKLB023 inhibits TGF- $\beta 1$-induced Smad2/3 phosphorylation in vitro}

We also assessed the capacity of SKLB023 to inhibit the iNOS expression of LX-2 by western blot analysis. As shown in Fig. 6, the protein expression of iNOS was significantly inhibited by SKLB023 in a dose-dependent manner.

It is well-known that TGF- $\beta 1$ is a major cytokine that induces the activation of HSCs via the activation of $\operatorname{Smad} 2 / 3 .^{23}$ To further explore the mechanism underlying the antifibrotic effect of SKLB023, the TGF- $\beta 1$-induced activation of Smad2/3 was evaluated in LX-2. As shown in Fig. 6, exposure to TGF- $\beta 1$ resulted in the significant upregulation of $\operatorname{Smad} 2 / 3$ phosphorylation compared to the control. SKLB023 treatment attenuated
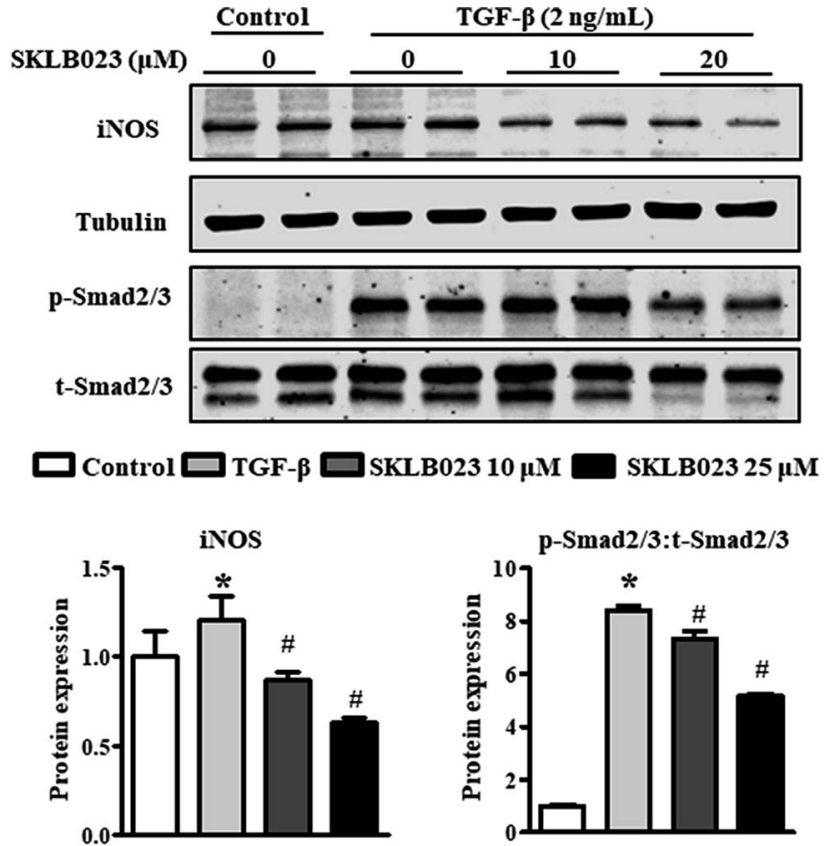

Fig. 6 SKLB023 inhibits TGF- $\beta 1$-induced Smad2/3 phosphorylation in vitro. TGF- $\beta 1$-induced LX-2 cells were treated with SKLB023 (10 and $20 \mu \mathrm{M})$ for $48 \mathrm{~h}$. The protein expressions of iNOS and $\mathrm{p}-\mathrm{Smad} 2 / 3$ were measured by western blot analysis, and the relative average protein level was determined by densitometry and normalized with $\beta$-tubulin or $\mathrm{t}-$ Smad2/3, respectively. ${ }^{*} P<0.05$ versus the control group, ${ }^{\#} P<$ 0.05 versus the vehicle.

the expression of TGF- $\beta 1$-induced Smad2/3 phosphorylation in a dose-dependent manner.

\section{Conclusions}

NASH is a progressive form of NAFLD. Unlike simple steatosis, NASH is characterized by hepatocellular injury accompanied by steatosis, inflammation and fibrosis. Although factors that might forecast the progression of NAFLD have not been clearly established, considerable studies have focused on the idea that initial fibrosis severity might be one of the most key prognostic factors. Therefore, preventing and treating liver fibrosis might be an optimal treatment for NAFLD. In this study, SKLB023, a potent small-molecule iNOS inhibitor, was confirmed to improve the progression of liver fibrosis. SKLB023 suppressed the activation of the TGF- $\beta$ /Smad signaling pathway by blocking iNOS expression to attenuate liver fibrosis in MCD diet-induced mice. These data suggest that SKLB023 is a novel and potential therapeutic strategy for antifibrotic intervention of NASH.

\section{Conflicts of interest}

There are no conflicts to declare.

\section{Acknowledgements}

The work was supported by the National Natural Science Foundation of China (81603035), China Postdoctoral 
Fellowship (2017M612981) and Postdoctoral Fellowship of Sichuan University (2017SCU12036).

\section{References}

1 P. Byass, BMC Med., 2014, 12, 159.

2 G. C. Farrell, D. van Rooyen, L. Gan and S. Chitturi, Gut Liver, 2012, 6, 149-171.

3 R. Bataller and D. A. Brenner, J. Clin. Invest., 2005, 115, 209218.

4 S. L. Friedman, Gastroenterology, 2008, 134, 1655-1669.

5 T. Higashi, S. L. Friedman and Y. Hoshida, Adv. Drug Delivery Rev., 2017, 121, 27-42.

6 T. Tsuchida and S. L. Friedman, Nat. Rev. Gastroenterol. Hepatol., 2017, 14, 397-411.

7 Y. Iwakiri and M. Y. Kim, Trends Pharmacol. Sci., 2015, 36, 524-536.

8 D. L. Diesen and P. C. Kuo, J. Surg. Res., 2011, 167, 96-112.

9 G. Aram, J. J. Potter, X. Liu, M. S. Torbenson and E. Mezey, Hepatology, 2008, 47, 2051-2058.

10 Y. Iwakiri, Clin. Mol. Hepatol., 2015, 21, 319-325.

11 E. Atik, Y. Onlen, L. Savas and F. Doran, Int. J. Infect. Dis., 2008, 12, 12-15.

12 S. J. Yoon, K. H. Choi and K. A. Lee, Mol. Reprod. Dev., 2002, 61, 504-510.
13 S. Anavi, M. Eisenberg-Bord, M. Hahn-Obercyger, O. Genin, M. Pines and O. Tirosh, Lab. Invest., 2015, 95, 914-924.

14 H. Kikuchi, T. Katsuramaki, K. Kukita, S. Taketani, M. Meguro, M. Nagayama, M. Isobe, T. Mizuguchi and K. Hirata, Wound Repair Regen., 2007, 15, 881-888.

15 L. Ma, C. Xie, Y. Ma, J. Liu, M. Xiang, X. Ye, H. Zheng, Z. Chen, Q. Xu, T. Chen, J. Chen, J. Yang, N. Qiu, G. Wang, X. Liang, A. Peng, S. Yang, Y. Wei and L. Chen, J. Med. Chem., 2011, 54, 2060-2068.

16 C. Xie, L. Ma, J. Liu, X. Li, H. Pei, M. Xiang and L. Chen, PLoS One, 2013, 8, e56349.

17 Y. Feng, J. Xu, F. Guo, R. Huang, M. Shi, L. Li, L. Ma and P. Fu, RSC Adv., 2018, 8, 5891-5896.

18 I. Zamin, Jr., A. A. Mattos, A. Z. Mattos, E. Migon, E. Soares and M. L. Perry, Arq. Gastroenterol., 2009, 46, 69-74.

19 C. Hellerbrand, B. Stefanovic, F. Giordano, E. R. Burchardt and D. A. Brenner, J. Hepatol., 1999, 30, 77-87.

20 Y. Tahashi, K. Matsuzaki, M. Date, K. Yoshida, F. Furukawa, Y. Sugano, M. Matsushita, Y. Himeno, Y. Inagaki and K. Inoue, Hepatology, 2002, 35, 49-61.

21 R. Derynck and Y. E. Zhang, Nature, 2003, 425, 577-584.

22 F. Xu, C. Liu, D. Zhou and L. Zhang, J. Histochem. Cytochem., 2016, 64, 157-167.

23 Y. Inagaki and I. Okazaki, Gut, 2007, 56, 284-292. 